\title{
OPEN Metformin hydrochloride entrapment in sorbitan monostearate for intestinal permeability enhancement and pharmacodynamics
}

\author{
OmarY. Mady ${ }^{1}$, Adam A. Al-Shoubki ${ }^{2 \bowtie}$, Ahmed A. Donia ${ }^{3}$ \& Waseem Qasim ${ }^{4}$
}

Penetration enhancement of metformin hydrochloride via its molecular dispersion in sorbitan monostearate microparticles is reported. This represents basic philosophy to maximize its entrapment for maximum penetration effect. Drug dispersion in sorbitan monostearate with different theoretical drug contents (TDC) were prepared. Products showed excellent micromeritics and actual drug content (ADC) increased by increasing TDC. The partition coefficient of the drug products showed huge improvement. This indicates the drug entrapped in the polar part of sorbitan monostearate as a special image which effects on the drug release. The drug permeation profiles from the different products are overlapped with nearly equal permeation parameters. The permeation results suggested the main driving force for improving the drug paracellular pathway is its dispersion in sorbitan monostearate and is independent of ADC. Pharmacodynamic of the products showed a significant improvement than the drug alone at $p<0.05$. ANOVA test indicated the insignificant pharmacodynamic difference between the low, middle, and high ADC of the products. An excellent correlation founded between the drug permeation and pharmacodynamic precents. Drug permeation driving force via the paracellular pathway is its entrapment in sorbitan monostearate and independent on ADC. The technique is simple and the products had excellent micromeritics.

Metformin Hydrochloride is one of the worldwide drugs used for the treatment of diabetic II patients. The drug is considered safe and does not produce a hypoglycemic effect ${ }^{1}$. It has some drawbacks concerned with gastrointestinal disorders especially with an older patient ${ }^{2}$. The marketed doses of the drug are 3 doses, 500, 850, and $1000 \mathrm{mg}$ per tablet with low bioavailability ${ }^{3}$. The biopharmaceutical classification system classifies metformin hydrochloride as a class III drug ${ }^{4}$. This group of drugs has high solubility but low intestinal permeability ${ }^{4}$. Then, it can understand the low bioavailability of the drug and consequently the high dose used in addition to the abdominal side effect.

Metformin hydrochloride was found to be absorbed (about 90\%) via the Paracellular pathway ${ }^{5,6}$. The high solubility of the drug and its Paracellular pathway absorption suggested a rapid and complete drug absorption. But the absorption of metformin hydrochloride is limited. It was reported that the addition of tween 80 to the drug led to an increase in all drug permeation parameters ${ }^{7,8}$. That is due to the role of non-ionic surfactants on enhancing metformin absorption via the paracellular pathway ${ }^{7,8}$. There is a significant saturable component of the paracellular pathway that limited the absorption of the highly water-soluble drug. The presence of tween 80 reduces the saturable paracellular pathway by electrostatic interactions between. the opposite charges of diffused substances (drug and tween 80 ) and the anionic residues of the lateral space and or tight junctions ${ }^{5,6,9,10}$. A novel "sponge" hypothesis was formulated to explain how metformin could be absorbed across human intestinal through the predominantly paracellular mechanism ${ }^{5,10}$.

\footnotetext{
${ }^{1}$ Pharmaceutical Technology Department, Faculty of Pharmacy, Tanta University, Tanta, Egypt. ${ }^{2}$ Pharmaceutics and Industrial Pharmacy Department, Faculty of Pharmacy, Omar Al-Mukhtar University, Al-Bayda, Libya. ${ }^{3}$ Department of Pharmaceutical Technology, Faculty of Pharmacy, Menoufia University, Shebeen El-Kom, Egypt. ${ }^{4}$ Department of Laboratory Sciences and Pharmaceutics, Faculty of Pharmacy, Ahl Al Bayt University, Karbala, Iraq. ${ }^{\circledR}$ email: adim.alsanousi@omu.edu.ly
} 
Mady et al. ${ }^{7}$ succeed to prepare sorbitan monostearate as microparticles containing metformin in the molecular state by using the rapid congealing technique. The molecular state of the drug in sorbitan monostearate was proved by different instrumental analyses. The authors suggested and used a modified non-everted sac technique in the discussion of the in-vitro drug permeation mechanism. They found that the addition of tween enhanced the drug permeation from the rabbit intestinal sac. The drug permeation enhancement was more pronounced from sorbitan monostearate entrapped drugs. The permeation parameters (permeation coefficient, total penetration percent and drug absorption enhancement \%) are markedly increased from sorbitan monostearate microparticles encapsulating drug. The authors concluded that, as a result of the drug polarity, the drug is encapsulated in the polar part of the surfactant which led to a huge increasing the encapsulated drug partition coefficient. Emulsification of sorbitan monostearate microparticles encapsulated drug in phosphate buffer $\mathrm{pH} 6.8$ by using tween 80 indicated the change of the surface again to hydrophilic. This image may be responsible for the above permeation parameters as a result of the correction of the HLP value (hydrophilic-lipophilic balances) of the image, which could be easily diffused through the paracellular pathway ${ }^{7}$.

Accordingly, the aim of this work is trying to investigate the penetration driving force of the drug via the paracellular pathway based on the previously reported by the author that, the drug dispersion in sorbitan monostearate has higher paracellular pathway penetration ${ }^{7}$. Then studying the relationship between increasing the actual drug content on the penetration effect to achieve the maximum drug dispersed in sorbitan monostearate for maximum penetration effect and consequently maximum pharmacodynamic effect. The pharmacodynamic study would be tested in diabetic rats. The micromeritics properties of the prepared products should be also studied, which are essential in the manufacture processing. At the end trying to find a correlation between the two normally related parameters for a drug, drug permeation enhancement percent and the drug pharmacodynamic enhancement percent.

\section{Materials and methods}

Materials. Metformin hydrochloride ( $\mathrm{HCl}$ ), was purchased from El-Nasr Pharmaceutical Chemical Co (Egypt), Sorbitan monostearate of research-grade was purchased from Altas Chemie, IC GmbH (Germany). All other chemicals were of analytical grade and used as received.

Methods. Dispersion of metformin $\mathrm{HCl}$ in sorbitan monostearate. Metformin $\mathrm{HCl}$ is dispersed in sorbitan monostearate by using the melting method. The required amounts of metformin $\mathrm{HCl}$ and sorbitan monostearate to prepare the solid dispersions of $25 \%, 33.33 \%, 50 \%, 66.66 \%$ and $75 \%$ theoretical drug content (TDC) in sorbi$\tan$ monostearate were weight and physically mixed ${ }^{11}$. The prepared physical mixtures were melted at $60^{\circ} \mathrm{C} \pm 5$ while stirring until clear molten solutions were obtained to assure from molecular dispersion of the drug in the carrier. The molten solutions were slowly cooled at room temperature while stirring to form sold masses. The products obtained were grinding, sieving, and stored at room temperature. The product particles, which passed from sieve size $600 \mu \mathrm{m}$ was used for further studies ${ }^{7,11}$.

Characterizations of the prepared drug-sorbitan monostearate solid dispersions. Determination of the actual drug content \% [ADC]. An accurate amount of each solid dispersion product containing $20 \mathrm{mg}$ as TDC was weighed and then dissolved in $100 \mathrm{ml}$ of $0.1 \mathrm{~N} \mathrm{HCl}$ at $60^{\circ} \mathrm{C}$. The solution may be filtered if necessary and measure spectrophotometry at $232 \lambda$ max using $0.1 \mathrm{HCl}$ as a blank. Sometimes dilutions may be carried out and the procedure was repeated in triplicate. The mean actual drug content and encapsulation \% were calculated using Eqs. (1) and (2) $)^{11,12}$.

$$
\begin{gathered}
\text { Theoretical drug content }(\mathrm{TDC})=\frac{\text { drug total }}{(\text { drug total }+ \text { sorbitan monostearate })} \times 100 \\
\text { Actual drug content }(\mathrm{ADC})=\frac{\text { Actual drug content total }}{(\text { drug total }+ \text { sorbitan monostearate })} \times 100
\end{gathered}
$$

Flow properties. The angle of repose method was used to study the follow property of the sorbitan monostearate-metformin solid dispersion products. The measuring was done by maintaining the funnel at a fixed height from a smooth glass surface in all experiments. The samples were passed through a funnel to form a stable cone ${ }^{13,14}$. The angle of repose ( $\Theta$ ) was calculated by using Eq. (3).

$$
\theta=\tan (\mathrm{h} / \mathrm{r})
$$

where $\theta=$ angle of repose, $h=$ height of cone, $r=$ radius of the cone base $e^{13,14}$.

Measurements of densities. A fixed weight of sorbitan monostearate-metformin solid dispersion product was carefully introduced into a $50 \mathrm{ml}$ graduated cylinder. The bulk volume (V0) was measured in $\mathrm{cm}^{3}$. Then dropping the cylinder from a high of $2.5 \mathrm{~cm}$ at one-second intervals. The procedure was repeated till no further change in volume was noted. The tapped volume $(\mathrm{Vt})$ was measured $\mathrm{cm}^{315-17}$. The densities parameters of the granules were calculated according to Eq. $(4)^{18}$, Eq. $(5)^{18}$, and Eq. $(6)^{19}$. 


$$
\begin{gathered}
\text { Bulk density }=\frac{\text { weight of the sample in gm }}{\text { volume in } \mathrm{cm}^{3}(\mathrm{~V} 0)} \\
\text { Tapped density }=\frac{\text { weight of the sample ingm }}{\text { volume in } \mathrm{cm}^{3}(\mathrm{Vt})} \\
\text { Carr's sindex }=\frac{\text { Tapped density }- \text { Bulk density }}{\text { Tapped density }} \times 100
\end{gathered}
$$

Experimentally determination of partition coefficients (Log P) by using n-octanol-water. A $20 \mathrm{mg}$ of either pure drug or that of each product containing $20 \mathrm{mg}$ metformin $\mathrm{HCl}$ determined from the actual drug content was taken and dissolved in $20 \mathrm{ml}$ of $\mathrm{n}$-octanol. Then $20 \mathrm{ml}$ of distilled water was added to the previous n-octanol solution while stirring. The formed system was transferred into a separating funnel and allowed to equilibrate. The concentration of the drug diffused from the organic phase (n-octanol) to the aqueous was measured spectrophotometrically at $232 \lambda$ max. using Eq. $(7)^{7,20}$

$$
\log \mathrm{p}=\log \left[\frac{\text { solute unionized in octanol }}{\text { solute ionized in water }}\right]
$$

Drug release profile. To the USP paddle dissolution apparatus, an accurate weight of the product containing $500 \mathrm{mg}$ of Metformin $\mathrm{HCl}$ [calculated according to the determined actual drug content] was added ${ }^{21}$. The release solution was $900 \mathrm{ml}$ of $0.1 \mathrm{~N} \mathrm{HCl}$ with a maintained temperature at $37 \pm 0.5^{\circ} \mathrm{C}$. The stirring rate was $100 \mathrm{rpm}$. The samples of $5 \mathrm{ml}$ were taken at predetermined time intervals for determining the cumulative drug release. A new release medium was added to replenish each sample withdrawn. Sometimes dilutions may be carried out and the resulting solutions were measured at $232 \lambda$ max using $0.1 \mathrm{HCl}$ as a blank. The procedure is carried out in triplicate ${ }^{7}$.

Non-everted sac model as a tool to evaluate the intestinal permeability. The method steps employed to evaluate the drug permeability profile from the modified non-everted sac method are modified experimental procedures described by references ${ }^{22-25}$.

- Preparation of non-everted intestinal sacs:

The animal used in this study was a Male albino rabbit with a weight of $2 \mathrm{~kg}$ obtained from the Tanta animal house. All procedures were approved and regularly controlled by the Animal Ethics Committee of Faculty of Pharmacy Tanta University (No: 2212018) and all experiments were performed by the guidelines and regulations of this committee. All the procedures were also carried out in full accordance with the ARRIVE guidelines $2020^{26}$, and adequate care was taken to minimize pain and discomfort for animals. Upon confirmation of loss of the pain reflex, the animal was sacrificed. A midline longitudinal incision of $3-4 \mathrm{~cm}$ was made, and the small intestine was located. A $14 \mathrm{~cm}$ segment of the intestine was used to prepare the sac. The lumen of the intestinal segment was washed with buffer to remove any solid material. Using a surgical thread, a side of the segment of the small intestine could be tied. The fresh intestinal sac was then filled with buffer, tied to the other side with surgical thread, and checked for leaks. The prepared segments after each step were placed in continuous aerated phosphate buffer and used for studying the drug permeation after filling with the perfusion solution ${ }^{7}$.

An amount of Sorbitan monostearate product containing $50 \mathrm{mg}$ of Metformin $\mathrm{HCl}$ as an actual drug content was accurately weighed and dispersed in $4 \mathrm{ml}$ of pH 6.8 phosphate buffer. One $\mathrm{ml}$ of tween 80 added. The fresh intestinal sac segment was then emptied from the buffer solution. The intestinal sac was then filled with prepared perfusion solution, tied with surgical thread, and tested for leaks. The segment length and diameter were measured for surface area determination ${ }^{7}$.

- Drug permeation profile study

The prepared segment was suspended on the shaft of the USP dissolution apparatus. The outside of the sac medium (permeation medium) was $900 \mathrm{ml}$ of phosphate buffer ( $\mathrm{pH}$ 6.8) with continuous aeration and maintained the temperature at $37 \pm 0.5^{\circ} \mathrm{C}$. The stirring rate was $50 \mathrm{rpm}$. Samples of $5 \mathrm{ml}$ were taken at predetermined time intervals and the new release medium was added to replenish each sample taken. The amount of drug permeated from the segment to the medium was determined spectrophotometrically at $232 \lambda \max ^{7}$.

- Determination of permeability coefficient

The use of Fick's law for the determination of the permeability coefficient (apparent permeability) of the drug across the isolated rat intestine was previously reported ${ }^{7,27,28}$. A simplified equation could be written as Eq. (8).

$$
\mathrm{dM} / \mathrm{dt}=\text { PSCd }
$$

The variables $\mathrm{M}$ and Cd could be determined by analysis of mucosal fluid. The surface area [S] could be calculated by considering the intestinal sac a cylinder. Then, M/SCd could be calculated and plotted against time. 


\begin{tabular}{|l|l|l|l|l|}
\hline TDC $\%$ & Compressibility $\%$ & \pm SD & Angle of repose & \pm SD \\
\hline 25 & 7.907 & 0.104 & 28.60 & 0.530 \\
\hline 33 & 6.009 & 0.165 & 29.14 & 0.082 \\
\hline 50 & 6.100 & 1.246 & 29.17 & 0.089 \\
\hline 66 & 7.872 & 1.254 & 29.85 & 0.681 \\
\hline 75 & 8.411 & 1.120 & 31.35 & 0.037 \\
\hline 100 & 26.767 & 0.910 & 39.71 & 0.550 \\
\hline
\end{tabular}

Table 1. Micromeritic properties of the prepared products.

The slope of the linear part of the plot is the permeability coefficient $(\mathrm{P})$, which has the units of velocity $(\mathrm{cm} / \mathrm{s})$. The slope of the linear part of the curve was determined by linear regression ${ }^{27-30}$.

Pharmacodynamics study.

- Animals

The animal used in this study was the male albino Wister rats aged 7-8 weeks with a weight of 150-200 g. All procedures were approved and regularly controlled by the Animal Ethics Committee of Faculty of Pharmacy Tanta University (No: 2212018) and all experiments were performed by the guidelines and regulations of this committee. All the procedures were also carried out in full accordance with the ARRIVE guidelines $2020^{26}$, and adequate care was taken to minimize pain and discomfort for animals. The housing of the animal was at an ambient temperature of $25 \pm 1{ }^{\circ} \mathrm{C}$ and relative humidity of $45-55 \%$ with a $12 \mathrm{~h}$ each of dark and light cycles. They fed was pellet diet and water ad libitum.

- Induction of the experimental diabetes

After overnight fasting of the animals, diabetes was induced by a single intraperitoneal injection of a freshly prepared solution of streptozotocin $\left(50 \mathrm{mg} / \mathrm{kg}\right.$ body weight) in $0.1 \mathrm{M}$ citrate buffer $(\mathrm{pH} 4.5)^{31,32}$. The animals were allowed to drink $5 \%$ glucose solution as a solution for the hypoglycaemic effect of the drug ${ }^{33}$. On the third day of streptozotocin injection, the rats fasted for $6 \mathrm{~h}$ and blood was withdrawn from the tail vein. The blood glucose level was measured by using Accu-Chek active (Accu-chek active test strip). Rats that had fasting blood glucose levels $>250 \mathrm{mg} / \mathrm{dl}$ were considered to be diabetic and were used to monitor the efficacy of metformin formulations ${ }^{34}$.

- Determination of the hypoglycaemic effect of metformin $\mathrm{HCl}$

For only $15 \mathrm{~min}$ on the day of the experiment, the rats were given free access to the pellet. To provide a stable blood glucose level, the food was restricted but the rats were given free access to water for $2 \mathrm{~h}$. Then, the formulations were dispersed in water at a concertation of $30 \mathrm{mg} / \mathrm{ml}$, and $0.125 \mathrm{ml}$ tween 80 was added for each $\mathrm{ml}$. From the prepared tested dispersion, $1 \mathrm{ml}$ was administered orally to each rat. At time intervals of $(0,0.25,0.5,1,2,3,4,5,6,7$, and 8$)$ hours, blood samples were withdrawn from the tail vein. The blood glucose was measured by using Accu-Chek active (Accu-chek active test strip). The blood glucose level was plotted as a function of time. The area above the curve was determined and used for monitoring the efficacy of different formulations. The amount of reduction in blood glucose level was also calculated and plotted as a function of time $e^{7,35}$.

- Statistical analysis

Statistical analysis of the in-vivo data was carried out by applying the ANOVA test. The data were analyzed by one-way analyses of variance (ANOVA) with blood glucose level as an independent factor.

Ethics approval. All procedures of animal study were approved and regularly controlled by the Animal Ethics Committee of Faculty of Pharmacy Tanta University (No: 2212018) and all experiments were performed in accordance with the guidelines and regulations of this committee. All the procedures were also carried out in full accordance with the ARRIVE guidelines $2020^{26}$.

\section{Results and discussion}

The work aims to determine the drug permeation driving force through the Paracellular pathway reported by the authors from sorbitan monostearate microparticles encapsulated the drug ${ }^{7}$. In that work, it was proved the molecular dispersion of the drug led to improving its Paracellular pathway permeation. To be assured from complete dispersion of the drug molecules and sorbitan monostearate, the solid dispersion is prepared by melting the physical mixture of the required amounts of the drug and the carrier. In addition, it could be expected there is no loss in both components of the solid dispersion as a result of the absence of a third component, which can dissolve either of the components or both. This led to assurance from nearly remaining the ratios of the components.

The effect of drug dispersion in the wax matrix on the drug processing could be noticed from the Table 1 . The flow property of all drug-matrices ratios was markedly improved from fair to passable flow in the case of the pure drug to very free-flowing except that prepared with 75\% TDC, which is free-flowing. That may be due to the low concentration of the wax matrix compared to other ratios. The closest of the angle of repose values on using different concentrations of the wax matrix may be due to the method of preparation, which is based on melting the drug and the matrix to form a clear melted solution before solid mass formation after cooling. Therefore, the 


\begin{tabular}{|l|l|l|}
\hline TDC $\%$ & ADC $\%$ & \pm SD \\
\hline 25 & 21.909 & 0.90 \\
\hline 33 & 31.455 & 1.29 \\
\hline 50 & 48.727 & 2.31 \\
\hline 66 & 64.909 & 1.03 \\
\hline 75 & 73.545 & 1.16 \\
\hline
\end{tabular}

Table 2. The actual (ADC) and theoretical (ATC) drug content of the prepared products.

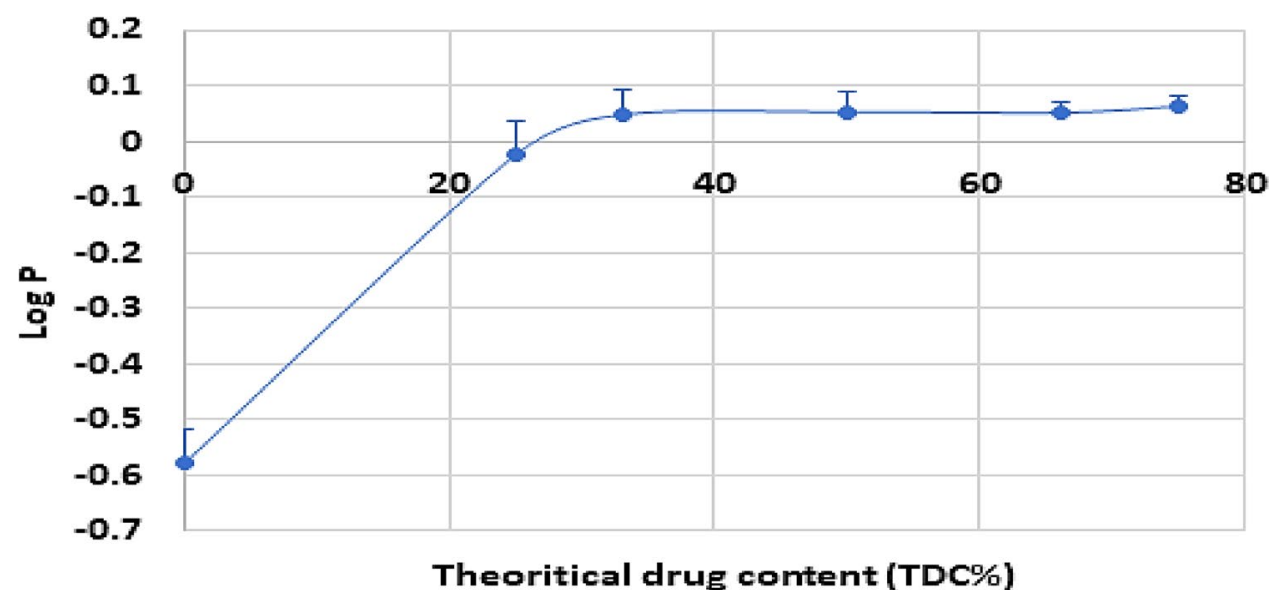

Figure 1. Effect of the theoretical drug content on its partition coefficient.

homogeneity of the dispersion of the drug in the dispersed substance could be expected. These results indicate the advantage of using sorbitan monostearate as a dispersed matrix since it improves the flowability of the dispersed drug which is sometimes considered as a limiting step in the pharmaceutical manufacture processes. In addition, to assure the homogeneity of using different concentrations of the dispersed media, it will be preferring to use the melting method to get a clear melt solution. This may be a disadvantage in using the melting technique for thermos-labile substances but it may be not considered because the melting point of sorbitan monostearate is around $56^{\circ} \mathrm{C}$. The compressibility index of the solid dispersed drug in sorbitan monostearate by using melting method is completely improved from poor compressible substance in case of the pure drug to excellent compressibility substance for all solid dispersed drug-sorbitan monostearate ratios ${ }^{36}$.

Since metformin-sorbitan monostearate solid dispersions were prepared by using melting technique, this led to supposes that there is no loss for both drug and matrix and the actual drug content should be equal to theoretical drug content. On the determination of the actual drug content, from a Table 2, it can be noticed that the actual drug content (ADC) is nearly equal to the theoretical drug content (TDC). The deviation of ACD from TDC is nearly constant and is maximum on using $25 \%$ TDC. That is maybe due to the insolubility of sorbitan monostearate in the extraction solution. ADC increased nearly parallel to increasing the TDC.

Partition coefficient. The partition coefficient of the pure drug and all drug solid dispersion products with different ratios were studied. Figure 1 showed that the partition coefficient of $25 \%$ drug dispersed in sorbitan monostearate as solid dispersion increased markedly than that of the pure drug. On using $33 \%$ drug in the solid dispersion form, the value of $\mathrm{Po} / \mathrm{w}$ is again increased and remains constant on increasing the drug percentage in the solid dispersion products. Since the drug-sorbitan monostearate solid dispersion is prepared by the melting method to produce a melt clear solution and the partition coefficient value depends on the lipophilicity of the drug, it can be concluded that the melting of the drug led to its dispersion in the polar part of the carrier. In this case, the polar part of the carrier could be only the polar part of an image of the surfactant which is, maybe, the surfactant micelle. This conclusion was also previously reported by the author ${ }^{7}$.

Dissolution profile. The drug release profile from different matrices prepared with different metforminspan ratios was studied. From Fig. 2, it can be noticed that there is rapid initial and incomplete drug release and both depend on the drug-matrix ratio. These two features are well-known and characterized for each drug dispersed in the molecular state in an insoluble matrix which is, maybe, also our case. Increasing the wax ratio led to decreasing the burst effect and total drug release and the reverse could be noticed concerning the drug. That is maybe due to the insolubility of sorbitan monostearate in the dissolution media. In addition, the high solubility of the drug in the dissolution media may enhance the burst effect. 


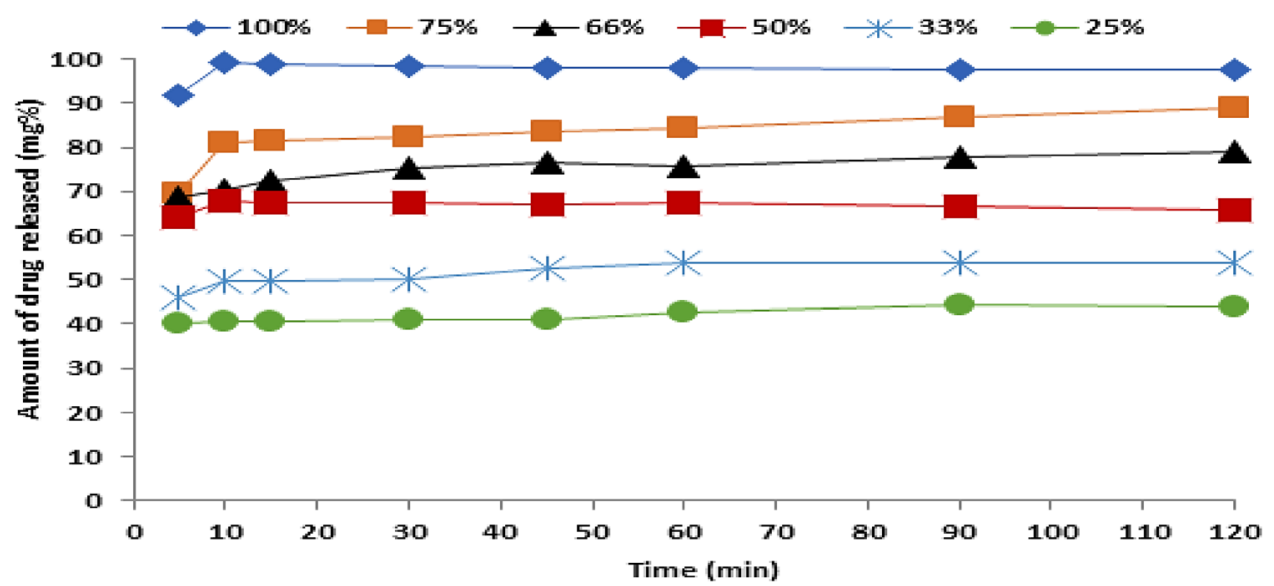

Figure 2. Drug release profile from different prepared drug-span matrices.

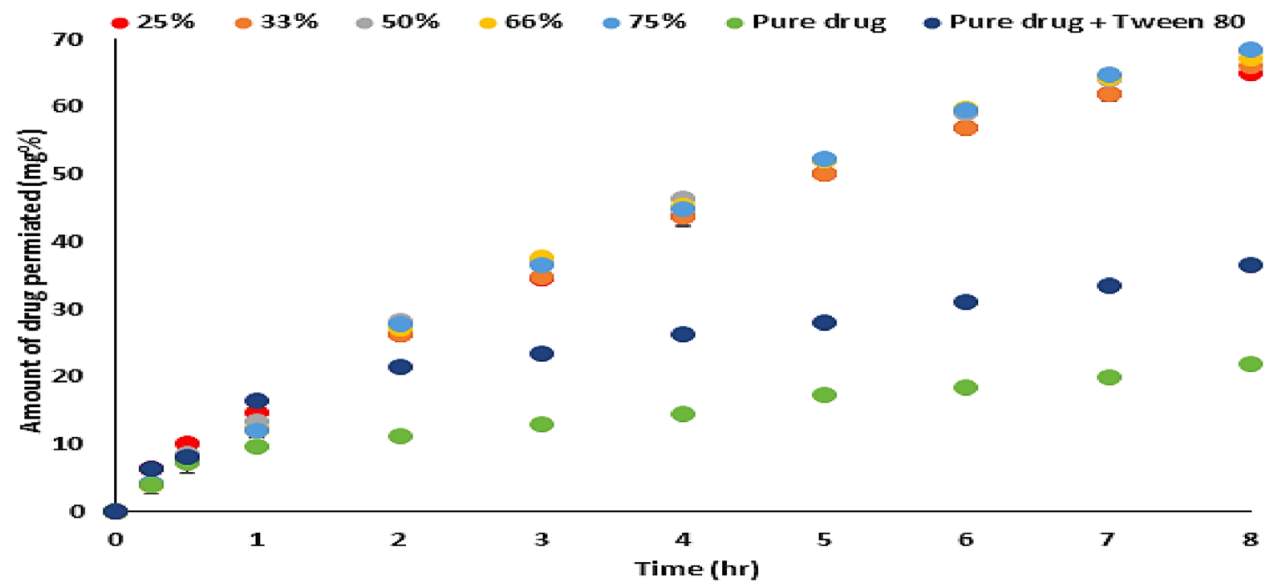

Figure 3. The permeability profiles of the pure drug, drug-tween, and different molecular solid dispersions of the drug in sorbitan monostearate matrices.

Permeation profile. The intestinal sacs for assaying the permeability are a quick and sensitive technique for determining the overall intestinal integrity or comparative transport of a specific molecule, with the added benefit of intestinal site-specificity. The apparent permeability [Papp] or permeation coefficient of a molecule through the intestinal barrier could be calculated ${ }^{37,38}$. The benefits of the application of the intestinal sac would be increased after solving the critical points facing its application by the author. Mady et al. ${ }^{7}$ succeed to solve the critical points of the technique by suspension the sac to the dissolution shaft in the dissolution media of the dissolution apparatus. This modification led to creating instead of drug release profile to drug permeation profile on using standard dissolution apparatus. The author discussed the critical points about the suggested solution, which may increase the value of the application of the technique as a modified non-everted sac.

Figure 3 showed that the addition of tween 80 to the pure drug enhances its permeability concerning the initial, rate and total drug permeated in the experimental time. That is due to different reported mechanisms $\mathrm{s}^{5,7,9,10}$ These effects would be more pronounced from the different solid dispersion products of the drug in sorbitan monostearate. The drug permeation profiles from the different drug-sorbitan monostearate solid dispersion products showed an overlap profile style concerning the initial, rate, and the total amount of drug released. This may be due to the drug dispersion in the carrier matrix and the use of an amount of the products containing the same actual drug concentration. Mady et al. ${ }^{7}$ discussed the essential of metformin HCL encapsulation in the molecular state in the carrier to improve its permeability. The conclusion of the author about the essential molecular dispersion of the drug in sorbitan monostearate as a carrier represents the basic philosophy to maximize its entrapment in the matrix. Increasing the initial drug permeated and total drug permeated leads to expecting improving the onset of action and decreasing the drug reported dose. Although metformin is reported to be safe from producing a hypoglycemic effect, decreasing the dose leads to decreasing the GIT side effect especially in older patients.

The permeability parameters of metformin $\mathrm{HCl}$, metformin-tween, and different dispersion of metforminsorbitan monostearate products across the non-everted sac were calculated and summarized in Table 3 . The values of $\mathrm{r}^{2}$, in each case, are high enough to consider a good fitting of the calculated permeation data. In every case, there is no lag time. In the case of metformin $\mathrm{HCl}$, the absences of lag time may be due to its high-water 


\begin{tabular}{|l|l|l|l|l|l|}
\hline & $\mathbf{r}^{\mathbf{2}}$ & Papp $(\mathbf{c m} / \mathbf{s}) \mathbf{1 0}^{-4}$ & Intercept & Total permeation \% & DAE \% \\
\hline Pure drug & 0.995 & 4.723 & 7.631 & $21.860( \pm 0.222)$ & 100.000 \\
\hline Drug-tween & 0.997 & 6.706 & 15.957 & $36.524( \pm 0.259)$ & 167.0814 \\
\hline 25\% span & 0.985 & 17.368 & 16.161 & $68.387( \pm 0.649)$ & 312.8408 \\
\hline $33 \%$ span & 0.973 & 17.644 & 16.975 & $67.032( \pm 0.432)$ & 306.6423 \\
\hline $50 \%$ span & 0.982 & 17.656 & 17.412 & $68.159( \pm 0.693)$ & 311.7978 \\
\hline $66 \%$ span & 0.984 & 17.764 & 15.205 & $65.972( \pm 1.061)$ & 301.7932 \\
\hline $75 \%$ Span & 0.979 & 18.239 & 15.586 & $64.960( \pm 0.400)$ & 297.1638 \\
\hline
\end{tabular}

Table 3. Metformin $\mathrm{HCl}$ transferred data through non-everted intestinal sac of pure drug, drug- tween, and different solid dispersions of the drug in sorbitan monostearate.

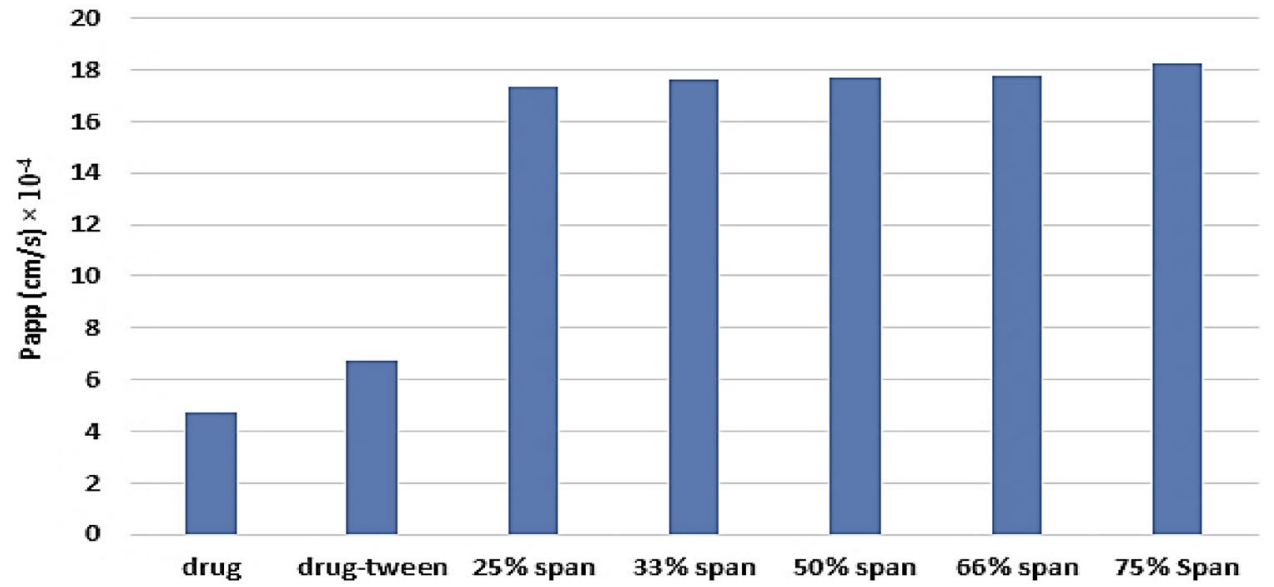

Figure 4. Histogram representation of the apparent permeability of metformin (M-to-S) from the pure drug, drug-tween, and drug-sorbitan monostearate solid dispersion products.

solubility which may be responsible for the presence of intercept values with y abscissa in concentration. The presence of an intercept value represents, in this case, the rapid saturation of the Paracellular pathway tissues of the intestinal wall with the drug before drug transport ${ }^{7}$. This finding is reinforced by the fact that about $90 \%$ of metformin $\mathrm{HCl}$ is absorbed via the Paracellular pathway ${ }^{5,6}$, and the absence of lag time. The addition of tween 80 to the drug led to increasing of all permeation parameters by different reported mechanisms $\mathrm{s}^{5,7,10}$. The permeation parameters of the drug from its different molecular dispersion products in sorbitan monostearate are markedly increased than that from drug-tween although the intercept values are nearly the same. In addition, the permeation parameters of the drug from its solid dispersion products prepared with different ratios are nearly equal which is reflected in the drug absorption enhancement (DAE \%). The drug absorption enhancement percent was calculated according to Eq. $(9)^{7}$

$$
\% \mathrm{DAE}=\frac{\text { The cumulative amount of drug penetrated from the dosage form }}{\text { The cumulative amount of pure drug penetrated }} \times 100
$$

From Table 3, it can be noticed that the drug absorption enhancing percent from different molecular drug solid dispersion percent are nearly equal.

Metformin $\mathrm{HCl}$ is a class III drug (highly water-soluble). Entrapment of the drug in sorbitan monostearate led to a huge increasing its partition coefficient indicating the entrapment of the drug occurred in the polar part of the surfactant. This led to the change of the drug from hydrophilic to the lipophilic entrapped image in sorbitan monostearate. The formed image led to the previously reported increasing the partition coefficient and decreasing the drug release. Emulsification of the image by tween 80 , led to a marked increasing the apparent permeability and consequently the total permeability of the drug. Emulsification of the image by tween 80 may be changed the image surface from lipophilic to hydrophilic and that may explain the absence of the lag time and the presence of the intercept with y abscissa in concentration. Changing the formed image surface to hydrophilic by adding tween 80 may be led to increasing the Paracellular pathway of the image-encapsulated drug. The similarity of the intercept values to the drug-tween indicating the same permeation mechanism of the image to the drug itself (paracellular pathway). These results could be also confirmed from the histogram representation of the total drug permeation Fig. 4.

The similarity of the drug permeation profiles, the permeability coefficient, total permeation percent, and drug absorption enhancement percent from its different solid dispersion products prepared with different ratios 
A

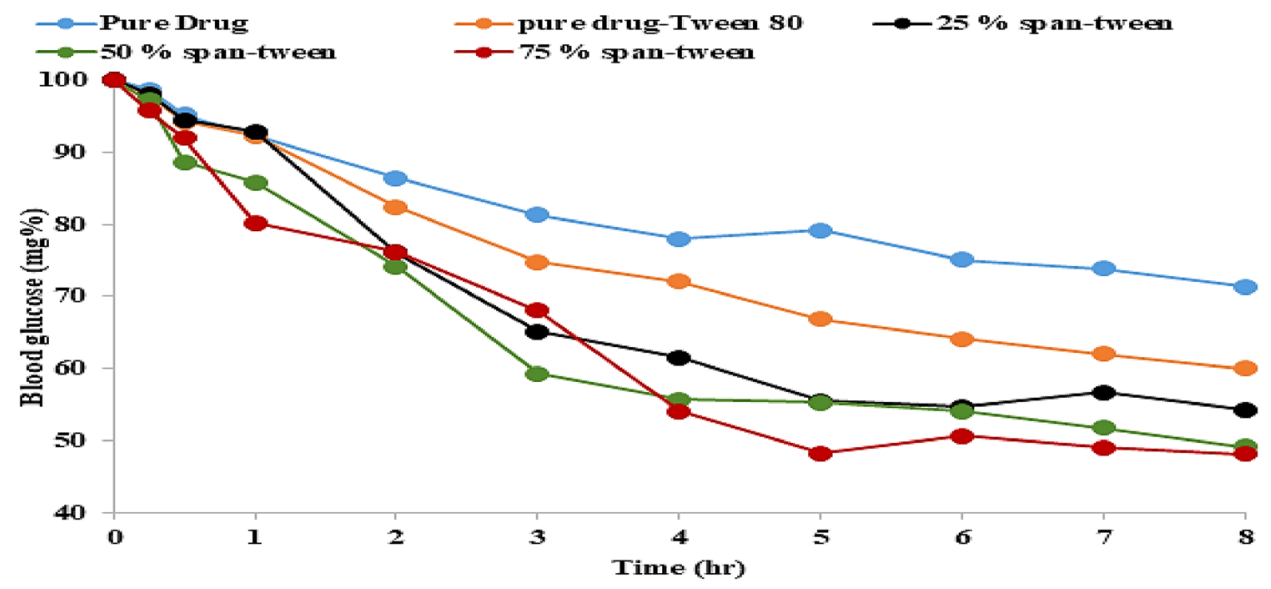

B
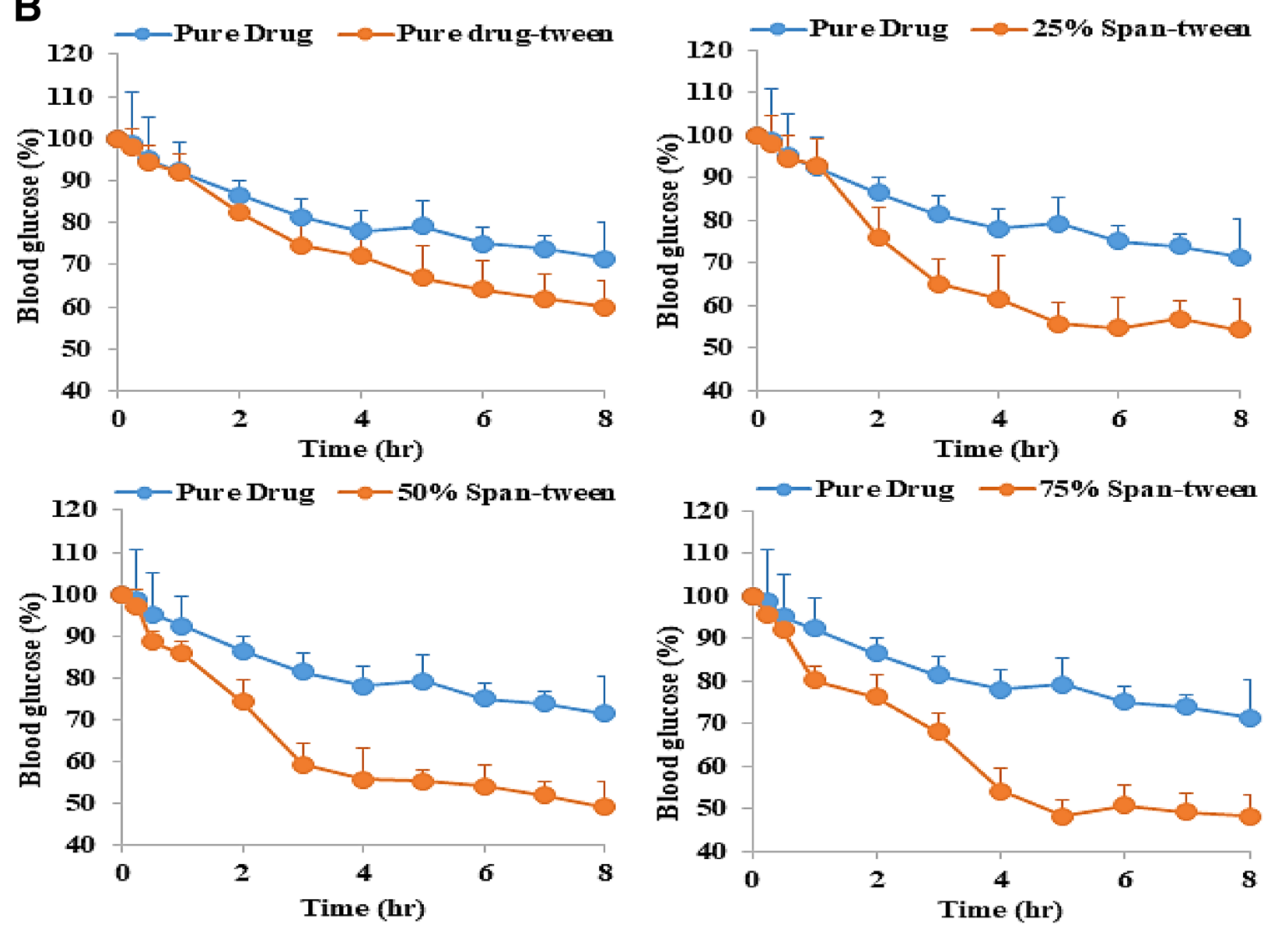

Figure 5. (A) Profiles of the change in glucose levels percent versus time. (B) Profiles of the change in glucose levels percent versus time (SD error bar).

suggesting that the role of sorbitan monostearate is only entrapment of the drug in its polar part as an image. This image entrapment process represents the drug permeation driving force, which is responsible for the paracellular permeation enhancement effect and this effect does not affect all drug-matrix ratios.

Pharmacodynamics evolution of the metformin $\mathrm{HCl}$ products. Evaluation of the in-vivo effect of metformin hydrochloride is monitored by using its pharmacodynamics marker parameter (lowering the blood glucose level after oral administration). The blood glucose level was measured before the drug administration, which represents the glucose level at zero time. After the drug administration, the plasma glucose level is monitored as a function of time. The blood glucose level would be expressed as a percent and the profile of drug glucose concentration is plotted as a function of time Fig. $5 \mathrm{~A}, \mathrm{~B}$.

A drop in the blood glucose level could be noticed from the first hour after oral drug administration. The effect of the high-water solubility of the drug and its paracellular pathway could be also reported as a result of the beginning of dropping of the blood glucose level after $15 \mathrm{~min}$. The maximum and rate of blood glucose level from drug-tween are higher than that from drug alone which confirms the results of the drug permeation through the modified permeation non everted sac test. Expected results could be noticed from the glucose dropping level as a result of administration of drug dispersed in sorbitan monostearate matrix. A clear significant difference 


\begin{tabular}{|l|l|l|l|l|l|}
\hline Reduction of blood glucose (mg/dl) & Drug & Drug/tween & $\mathbf{2 5 \%}$ & $\mathbf{5 0} \%$ & $\mathbf{7 5 \%}$ \\
\hline Time (h) & 0.0 & 0.0 & 0.0 & 0.0 & 0.0 \\
\hline 0.0 & $9.75( \pm 57.8)$ & $10.75( \pm 23.0)$ & $10.6( \pm 33.7)$ & $15.0( \pm 20.1)$ & $22.0( \pm 6.5)$ \\
\hline 0.25 & $16.75( \pm 42.1)$ & $18.25( \pm 5.7)$ & $18.6( \pm 60.9)$ & $44.0( \pm 28.6)$ & $19.0( \pm 9.6)$ \\
\hline 0.5 & $13( \pm 37.4)$ & $10.25( \pm 20.1)$ & $8.3( \pm 7.6)$ & $14.3( \pm 7.5)$ & $59.3( \pm 5.1)$ \\
\hline 1.0 & $28.25( \pm 20.0)$ & $49.25( \pm 35.0)$ & $86.6( \pm 66.4)$ & $59.3( \pm 27.4)$ & $20.0( \pm 10.5)$ \\
\hline 2.0 & $25.25( \pm 12.5)$ & $38.25( \pm 14.2)$ & $56.6( \pm 9.5)$ & $76.0( \pm 6.6)$ & $41.7( \pm 8.1)$ \\
\hline 3.0 & $16.25( \pm 9.0)$ & $13( \pm 21.1)$ & $20.3( \pm 49.0)$ & $19.0( \pm 24.1)$ & $70.3( \pm 9.1)$ \\
\hline 4.0 & $-5.3( \pm 23.1)$ & $26.5( \pm 46.2)$ & $28.3( \pm 72.6)$ & $1.3( \pm 37.2)$ & $30.0( \pm 15.4)$ \\
\hline 5.0 & $19.5( \pm 16.0)$ & $13.25( \pm 33.0)$ & $5( \pm 37.0)$ & $6.3( \pm 17.2)$ & $-12.7( \pm 9.9)$ \\
\hline 6.0 & $6( \pm 6.4)$ & $10.5( \pm 17.7)$ & $-11.0( \pm 35.5)$ & $11.7( \pm 12.3)$ & $8.3( \pm 4.0)$ \\
\hline 7.0 & $13.75( \pm 31.1)$ & $10( \pm 16.2)$ & $14.6( \pm 55.1)$ & $14.0( \pm 25.0)$ & $4.3( \pm 7.1)$ \\
\hline 8.0 & $882.63( \pm 231.4)$ & $1067.8( \pm 115.9)$ & $1355.8( \pm 164.2)$ & $1473.7 \pm 185.2)$ & $1512.0( \pm 141.9)$ \\
\hline AAC (mg h/dl) & & 20.98 & 53.61 & 66.97 & 71.31 \\
\hline Pharmacodynamic enhancement \% & & & & $63.96( \pm 9.23)$ & \\
\hline Mean pharmacodynamic enhancement \% & & & & & \\
\hline
\end{tabular}

Table 4. The Data of the reduction of blood glucose level and the area above the blood glucose level versus time curve, obtained after oral administration of different Metformin $\mathrm{HCl}$ products to diabetic rats. Values between brackets are S.E.M. $(n=6)$.

between the pharmacodynamic effect of the pure drug and the dropping of glucose level after administration of lower, middle, or higher drug entrapped in sorbitan monostearate at $\mathrm{p}<0.05$ on applying ANOVA test was found.

From Fig. 5A, it can be noticed that the dropping of glucose level profile after administration of lower (25\%), middle (50\%), and higher (75\%) drug entrapped in sorbitan monostearate are intersecting at more than one point to the degree of congruence. Applying the ANOA test concluded there is no significant difference between the pharmacodynamic effect (dropping of glucose level) of the three products at $p<0.05$, which confirms again the drug permeation results from the modified non-everted sac technique. It should be reported that each point represents the mean of 6 blood glucose level measurements at that time with standard deviation as shown in Fig. 5B.

The area above the curves (AAC) was calculated from Fig. 5. The results are tabulated in Table 4. Drug pharmacodynamic enhancement percent was calculated according to Eq. (10).

$$
\text { Drug pharmacodynamic enhancement } \%=\frac{\text { (AAC of treated drug }- \text { AAC of pure drug) }}{(\text { AAC of pure drug })} \times 100
$$

From Table 4, it can be noticed that the drug pharmacodynamic enhancement \% increased according to the following order: $75 \%{ }^{>} 50 \%$ ' $25 \%$ 'drug-tween. The mean of pharmacodynamic enhancement percent is $64 \%$, which is equal to drug absorption enhancement effect $\%$ as a result of drug molecular dispersion in sorbitan monostearate Table 3.

Drug intestinal permeation-pharmacodynamic correlation. FDA defined in-vitro-in-vivo correlation (IVIVC) as "a predictive mathematical model describing the relationship between an in vitro property of a dosage form and a relevant in vivo response". In general, the in vitro property is the rate or extent of drug dissolution or release while the in vivo response is the plasma drug concentration or amount of drug absorbed ${ }^{39-42}$. FDA guidance described 4 levels for IVIVC which are A, B, C, and multiple $\mathrm{C}^{40}$. In this study, level A was selected because of its highest category of correlation since it correlates a point-to-point relationship ${ }^{41,42}$.

The pharmacodynamic marker of metformin is its dropping effect on the blood glucose level. Therefore, it was tried to correlate (point-to-point correlation) the percentage of drug permeated and its dropping of blood glucose level percentage. From Fig. 6A-E, it can be noticed that the drug permeation percent curve is the opposite superimposed to the blood glucose dropping percent curve. That is maybe due to the glucose dropping effect is dependent on the blood drug concentration. The area between the two curves would be decreased by increasing the percent of drug molecular dispersed in the span matrix until intercepted at the special point.

Since Level A correlation is a linear relationship between two variables, it was tried to create a mathematic line correlation between the drug permeation enhancing percent and its pharmacodynamics enhancing percent (dropping of glucose level) as a point-to-point correlation. Table 5 shows the value of the correlation coefficient in every case is high enough to conclude a linear correlation between the drug permeation enhancing percent and its pharmacodynamic enhancing percent. Decreasing the slope values of the products than from both drug alone and drug plus tween confirm the drug penetration enhancements, which is consequently lead to more dropping of blood glucose level. The nearly similar slope values of the low, middle, and high drug entrapment products are in agreement with the insignificant difference in the drug permeability and pharmacodynamic effect between the three products. In addition, the results supported the conclusion about the drug permeation driving force is its molecular dispersion in the sorbitan monostearate suggesting image, which is not dependent on the percentage of drug entrapped. Decreasing the intercept values of the products from the pure drug may be, 

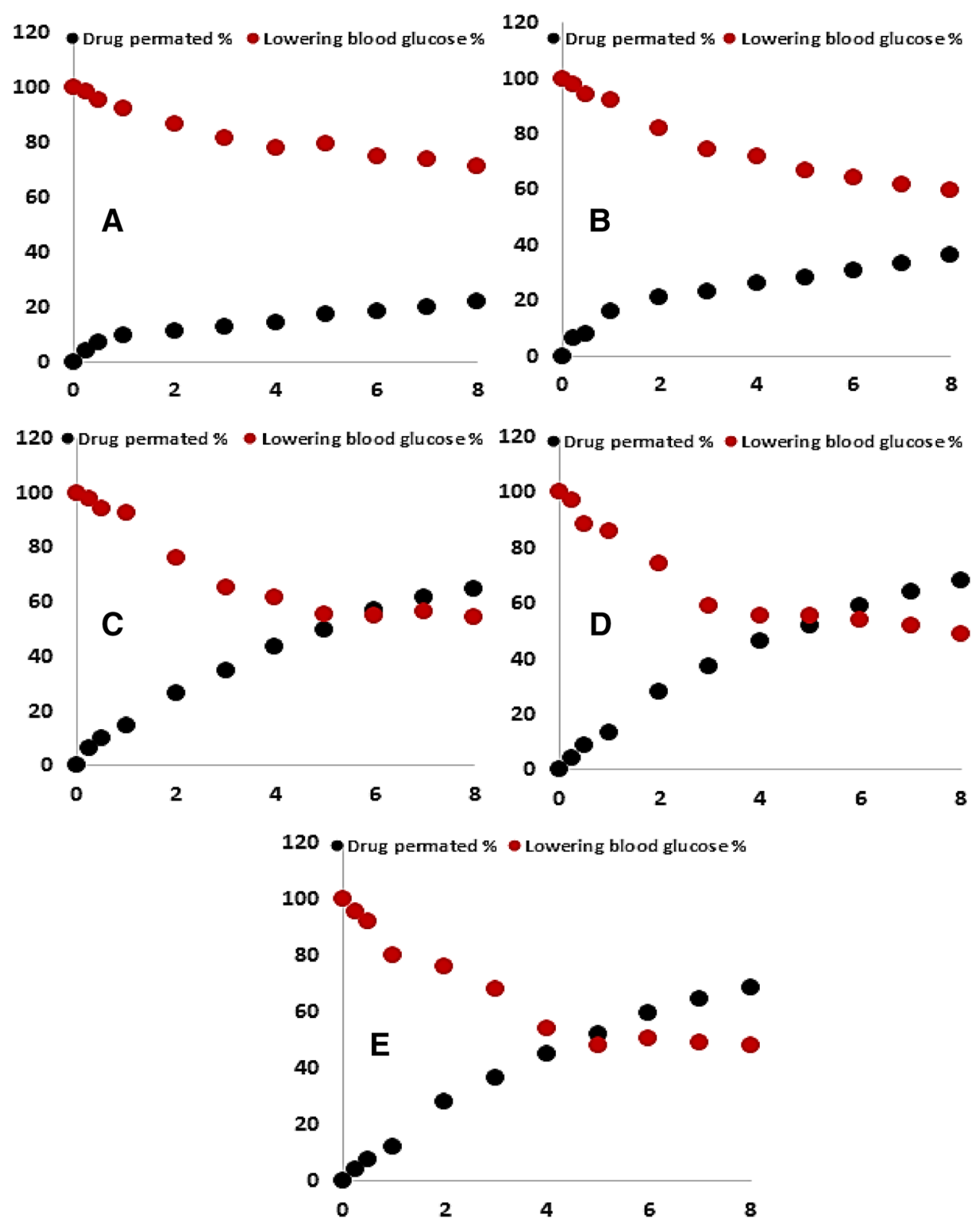

Figure 6. Point to point (level A) correlation of the drug permeation profiles and its pharmacodynamics effect: (A) metformin HCL; (B) metformin-tween 80; (C) 25\% drug in span-tween. (D) 50\% drug in span-tween; (E) $75 \%$ drug in span-tween.

\begin{tabular}{|l|l|l|l|}
\hline & $\mathbf{r}^{2}$ & Slope & Intercept \\
\hline Metformin HCl & 0.948 & -1.471 & 102.89 \\
\hline Metformin plus tween 80 & 0.950 & -1.237 & 104.73 \\
\hline 25\% metformin SD & 0.944 & -0.791 & 100.11 \\
\hline $50 \%$ metformin SD & 0.951 & -0.755 & 96.231 \\
\hline $75 \%$ metformin SD & 0.949 & -0.783 & 96.162 \\
\hline
\end{tabular}

Table 5. Correlation data of drug permeation and pharmacodynamics profile:

indicates the unstatutable paracellular pathway of the drug absorption from the suggested image, which facing the pure drug absorption.

From the above study, it was found the following: 1. the drug permeation profiles from all drug molecular dispersed products in sorbitan monostearate (low, middle and high) are found to be overlapped to each other. 2. The drug pharmacodynamic effect of the prepared products is intersecting at more than one point to the degree of 


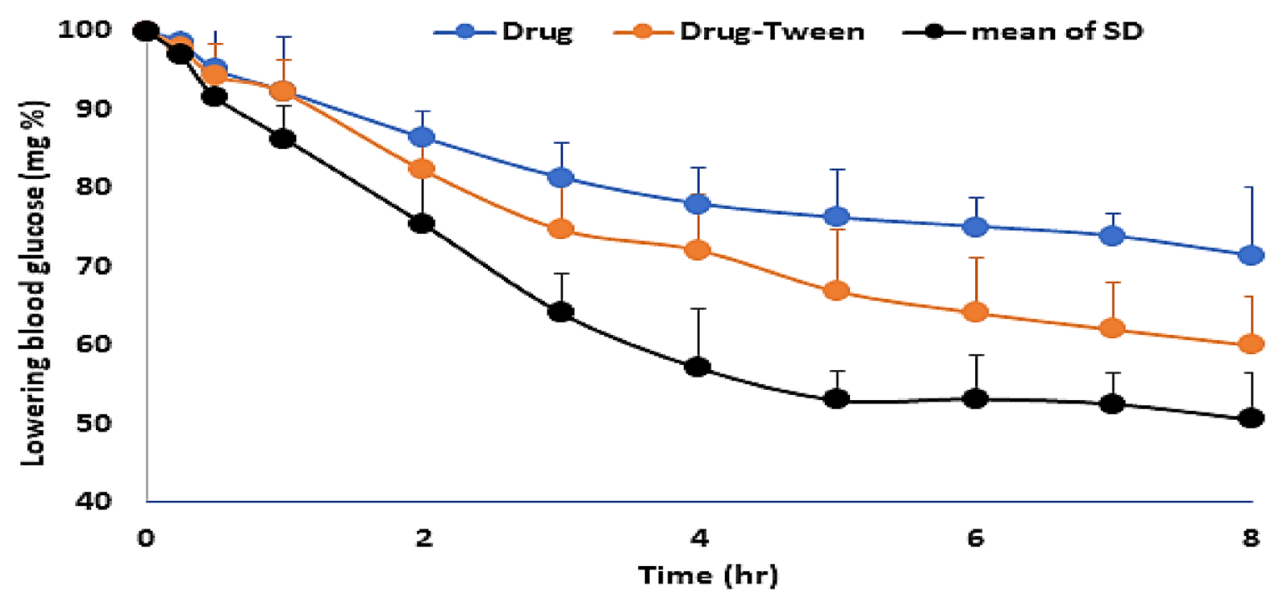

Figure 7. Profiles of the change in glucose levels percent versus time (SD error bar).

congruence. 3. Applying the ANOA test showed there is no significant difference between the pharmacodynamic effect (dropping of glucose level) of the three-drug molecular dispersed products at $p<0.05$. 4. The mean drug absorption (permeation) percent is equal to the mean pharmacodynamic percent. Accordingly, it can be reported that the paracellular enhancement of sorbitan monostearate to metformin is based on its dispersion in the matrix, which is confirmed by the author before ${ }^{7}$, and the enhancement does not depend on the drug-matrix ratio. This conclusion is supported by the results of the ANOVA test, which shows no significant difference between the drug products' pharmacodynamic effects. Accordingly, the mean pharmacodynamic effect of the low, middle and high drug molecular dispersed in the matrix was calculated and is represented in Fig. 7 with bar standard deviation.

\section{Conclusion}

From this study, it can be concluded that the permeability problem facing metformin, as an example for the class III drugs according to BSC classification, may be solved by its dispersion in sorbitan monostearate. Sorbitan monostearate is widely used in food industries and its uses in pharmaceutical technology would be harmless. The high hydrophilicity property of the Class III drug solubilizes them in the polar part of sorbitan monostearate in a special image, which may be theoretically micelle form. The image suggested by the authors (micelle) represents the penetration driving force of class III group drugs via the paracellular pathway. This image, which needs more investigation, does not depend on the drug-matrix ratio. Permeability enhancement could also test by using the author's suggested modified non-everted sac technique. This conclusion is based on an excellent relationship found between the drug permeation percent and its pharmacodynamic effect percent. Improving drug permeability leads to improving the drug bioavailability and consequently leads to decreasing the drug dose and side effects to the patient. At the same time decreasing the cost for the pharmaceutical industry as a result of decreasing the raw materials required, in addition to the simplicity and reproducibility of the technique used, encourage to suggest the technique for its dual effects.

\section{Data availability}

The raw data supporting the conclusions of this manuscript will be made available by the authors, without undue reservation, to any qualified researcher.

Received: 14 June 2021; Accepted: 24 September 2021

Published online: 11 October 2021

\section{References}

1. Yendapally, R. et al. A review of phenformin, metformin, and imeglimin. Drug Dev. Res. 81(4), 390-401. https://doi.org/10.1002/ ddr.21636 (2020).

2. Mancer, D., Allemann, E. \& Daoud, K. Metformin hydrochloride microencapsulation by complex coacervation: Study of size distribution and encapsulation yield using response surface methodology. J. Drug Deliv. Sci. Technol. 45, 184-195. https://doi.org/ 10.1016/j.jddst.2018.03.015 (2018).

3. WookHuh, H. et al. Novel self-floating tablet for enhanced oral bioavailability of metformin based on cellulose. Int. J. Pharm. 592, 120113. https://doi.org/10.1016/j.ijpharm.2020.120113 (2021).

4. Umeta, B. et al. Dissolution profile evaluation of eight brands of metformin hydrochloride tablets available in Jimma, Southwest Ethiopia. Diabetes Metab. Syndrome Obes. Targets Ther. 14, 3499-3506. https://doi.org/10.2147/dmso.s316187 (2021).

5. Proctor, W. R., III: A Novel Mechanism for Intestinal Absorption of the Type II Diabetes Drug Metformin: Role of Cation-selective Apical Transporters in Paracellular Absorption, Chapel Hill 2010.

6. Saitoh, R. et al. Correction of permeability with pore radius of tight junctions in Caco-2 monolayers improves the prediction of the dose fraction of hydrophilic drugs absorbed by humans. Pharm. Res. 21(5), 749-755. https://doi.org/10.1023/b:pham.00000 26423.48583.e2 (2004).

7. Mady, O. Y., Donia, A. A., Al-Shoubki, A. A. \& Qasim, W. Paracellular pathway enhancement of metformin hydrochloride via molecular dispersion in span 60 microparticles. Front. Pharmacol. https://doi.org/10.3389/fphar.2019.00713 (2019). 
8. Jin, S. et al. Enhanced intestinal permeability and plasma concentration of metformin in rats by the repeated administration of red Ginseng extract. Pharmaceutics 11(4), 189. https://doi.org/10.3390/pharmaceutics11040189 (2019).

9. Alvi, M. M. \& Chatterjee, P. A prospective analysis of co-processed non-ionic surfactants in enhancing permeability of a model hydrophilic drug. AAPS PharmSciTech 15(2), 339-353. https://doi.org/10.1208/s12249-013-0065-8 (2013).

10. Proctor, W. R., Bourdet, D. L. \& Thakker, D. R. Mechanisms underlying saturable intestinal absorption of metformin. Drug Metab. Dispos. 36(8), 1650-1658. https://doi.org/10.1124/dmd.107.020180 (2008).

11. Mady, O. Span 60 as a microsphere matrix: Preparation and in vitro characterization of novel ibuprofen-span 60 microspheres. J. Surfact. Deterg. 20(1), 219-232. https://doi.org/10.1007/s11743-016-1907-7 (2016).

12. Mao, S. et al. Effects of process and formulation parameters on characteristics and internal morphology of poly(d, l-lactide-coglycolide) microspheres formed by the solvent evaporation method. Eur. J. Pharm. Biopharm. 68(2), 214-223. https://doi.org/10. 1016/j.ejpb.2007.06.008 (2008).

13. Guo, Z. et al. Theoretical and experimental investigation on angle of repose of biomass-coal blends. Fuel 116, 131-139. https:// doi.org/10.1016/j.fuel.2013.07.098 (2014).

14. Allenspach, C., Timmins, P., Sharif, S. \& Minko, T. Characterization of a novel hydroxypropyl methylcellulose (HPMC) direct compression grade excipient for pharmaceutical tablets. Int. J. Pharm. 583, 119343. https://doi.org/10.1016/j.ijpharm.2020.119343 (2020).

15. Batra, A., Thongsukmak, A., Desai, D. \& Serajuddin, A. T. M. The effect of process variables and binder concentration on tabletability of metformin hydrochloride and acetaminophen granules produced by twin screw melt granulation with different polymeric binders. AAPS PharmSciTech 22, 4. https://doi.org/10.1208/s12249-021-02018-6 (2021).

16. E Silva, J. P. S., Splendor, D., Gonçalves, I. M. B., Costa, P. \& Sousa Lobo, J. M. Note on the measurement of bulk density and tapped density of powders according to the European Pharmacopeia. AAPS PharmSciTech 14(3), 1098-1100. https://doi.org/10.1208/ s12249-013-9994-5 (2013).

17. Aguilar-Díaz, J. E. et al. Predicting orally disintegrating tablets formulations of ibuprophen tablets: An application of the new SeDeM-ODT expert system. Eur. J. Pharm. Biopharm. 80(3), 638-648. https://doi.org/10.1016/j.ejpb.2011.12.012 (2012).

18. Reddy, K. R., Mutalik, S. \& Reddy, S. Once-daily sustained-release matrix tablets of nicorandil: Formulation and in vitro evaluation. AAPS PharmSciTech 4(4), 480-488. https://doi.org/10.1208/pt040461 (2003).

19. Youness, D., Hosseinpoor, M., Yahia, A. \& Tagnit-Hamou, A. Flowability characteristics of dry supplementary cementitious materials using Carr measurements and their effect on the rheology of suspensions. Powder Technol. 378, 124-144. https://doi.org/10. 1016/j.powtec.2020.09.064 (2021).

20. Farag, M. M., Abd El Malak, N. S., Yehia, S. A. \& Ahmed, M. A. Sonocomplexation as an effective tool to enhance the antitumorigenic effect of metformin: Preparation, in vitro characterization, molecular dynamic simulation \& MiaPaCa-2 cell line hypoxia evaluation. J. Drug Deliv. Sci. Technol. 59, 101968. https://doi.org/10.1016/j.jddst.2020.101968 (2020).

21. Pharmacopoeia, B. Version 11.0. Appendices: XII A, XII G, XVII G. https://doi.org/10.1016/b978-0-7506-6878-1.50004-9 (2007).

22. Alshamsan, A., Kazi, M., Badran, M. M. \& Alanazi, F. K. Role of alternative lipid excipients in the design of self-nanoemulsifying formulations for fenofibrate: Characterization, in vitro dispersion, digestion and ex vivo gut permeation studies. Front. Pharmacol. https://doi.org/10.3389/fphar.2018.01219 (2018).

23. Freag, M. S., Elnaggar, Y. S. R. \& Abdallah, O. Y. Development of novel polymer-stabilized diosmin nanosuspensions: In vitro appraisal and ex vivo permeation. Int. J. Pharm. 454(1), 462-471. https://doi.org/10.1016/j.ijpharm.2013.06.039 (2013).

24. Jha, S. K., Karki, R., Puttegowda, V. D. \& Harinarayana, D. In vitro intestinal permeability studies and pharmacokinetic evaluation of famotidine microemulsion for oral delivery. Int. Schol. Res. Not. 2014, 1-7. https://doi.org/10.1155/2014/452051 (2014).

25. Shishu, X. \& Maheshwari, M. Comparative bioavailability of curcumin, turmeric and Biocurcumax ${ }^{\mathrm{TM}}$ in traditional vehicles using non-everted rat intestinal sac model. J. Funct. Foods 2(1), 60-65. https://doi.org/10.1016/j.jff.2010.01.004 (2010).

26. PercieduSert, N. et al. Reporting animal research: Explanation and elaboration for the ARRIVE guidelines 20. PLoS Biol. 18(7), e3000411. https://doi.org/10.1371/journal.pbio.3000411 (2020).

27. Friedrich, M. Membrane transport in biology. Herausgegeben von G. Giebisch, D. C. Tosteson und H. H. Ussing. Vol. 3. Transport Across Multi-Membrane Systems. Herausgegeben von G. Giebisch. XVIII und 459 Seiten. 97 Abb., 26 Tab. Springer-Verlag, Berlin, Heidelberg, New York 1978. Preis: 148,- DM; 81,40 \$. Food Nahrung 24(2), 202-202. https://doi.org/10.1002/food.19800240214 (1980).

28. Page, D. A. \& Carlson, G. P. Method for studying the permeability of the rat intestinal tract to carbon tetrachloride. Toxicol. Methods 1(3), 188-198. https://doi.org/10.3109/15376519109044569 (1991).

29. Watanabe, E., Takahashi, M. \& Hayashi, M. A possibility to predict the absorbability of poorly water-soluble drugs in humans based on rat intestinal permeability assessed by an in vitro chamber method. Eur. J. Pharm. Biopharm. 58(3), 659-665. https:// doi.org/10.1016/j.ejpb.2004.03.029(2004).

30. Ye, J. et al. Comparisons of in vitro Fick's first law, lipolysis, and in vivo rat models for oral absorption on BCS II drugs in SNEDDS. Int. J. Nanomed. 14, 5623-5636. https://doi.org/10.2147/ijn.s203911 (2019).

31. Sayeli, V. K. \& Shenoy, A. K. Antidiabetic effect of bio-enhanced preparation of turmeric in streptozotocin-nicotinamide induced type 2 diabetic Wistar rats. J. Ayurveda Integrat. Med. 12(3), 474-479. https://doi.org/10.1016/j.jaim.2021.04.010 (2021).

32. Bathina, S. et al. Resolvin D1 ameliorates nicotinamide-streptozotocin-induced type 2 diabetes mellitus by its anti-inflammatory action and modulating PI3K/Akt/mTOR pathway in the brain. Arch. Med. Res. 51(6), 492-503. https://doi.org/10.1016/j.arcmed. 2020.05.002 (2020).

33. Harley, B. K. et al. Myrianthus libericus: Possible mechanisms of hypoglycaemic action and in silico prediction of pharmacokinetics and toxicity profile of its bioactive metabolite, friedelan-3-one. Biomed. Pharmacother. 137, 111379. https://doi.org/10.1016/j. biopha.2021.111379 (2021).

34. Ravi, K., Ramachandran, B. \& Subramanian, S. Protective effect of Eugenia jambolana seed kernel on tissue antioxidants in streptozotocin-induced diabetic Rats. Biol. Pharm. Bull. 27(8), 1212-1217. https://doi.org/10.1248/bpb.27.1212 (2004).

35. Hemalatha, S., Wahi, A., Singh, P. \& Chansouria, J. P. Hypoglycemic activity of Withania coagulans Dunal in streptozotocin induced diabetic rats. J. Ethnopharmacol. 93(2-3), 261-264. https://doi.org/10.1016/j.jep.2004.03.043 (2004).

36. United States Pharmacopeial Convention. United States pharmacopeia 38 national formulary 33 . https://doi.org/10.32388/2yh02t (2016).

37. Xu, Y., Shrestha, N., Préat, V. \& Beloqui, A. An overview of in vitro, ex vivo and in vivo models for studying the transport of drugs across intestinal barriers. Adv. Drug Deliv. Rev. 175, 113795. https://doi.org/10.1016/j.addr.2021.05.005 (2021).

38. Jacobsen, A.-C., Nielsen, S., Brandl, M. \& Bauer-Brandl, A. Drug permeability profiling using the novel Permeapad ${ }^{\circ} 96-$ well $^{2}$ plate. Pharm. Res. 37, 6. https://doi.org/10.1007/s11095-020-02807-x (2020).

39. Li, X., Zhao, Z., Li, L., Zhou, T. \& Lu, W. Pharmacokinetics, in vitroandin vivocorrelation, and efficacy of exenatide microspheres in diabetic rats. Drug Deliv. 22(1), 86-93. https://doi.org/10.3109/10717544.2013.871760 (2014).

40. Saibi, Y., Sato, H. \& Tachiki, H. Developing in vitro-in vivo correlation of risperidone immediate release tablet. AAPS PharmSciTech 13(3), 890-895. https://doi.org/10.1208/s12249-012-9814-3 (2012).

41. Sakai, M., Imai, T., Ohtake, H., Azuma, H. \& Otagiri, M. Effects of absorption enhancers on the transport of model compounds in Caco-2 cell monolayers: Assessment by confocal laser scanning microscopy. J. Pharm. Sci. 86(7), 779-785. https://doi.org/10. 1021/js960529n (1997). 
42. Lo, Y. Relationships between the hydrophilic-lipophilic balance values of pharmaceutical excipients and their multidrug resistance modulating effect in Caco-2 cells and rat intestines. J. Control. Release 90(1), 37-48. https://doi.org/10.1016/s0168-3659(03) 00163-9 (2003).

\section{Acknowledgements}

We have to express our appreciation to workers and faculty members, the Department of Pharmaceutical Technology, Faculty of Pharmacy, Tanta University for sharing their pearls of wisdom with us during this research.

\section{Author contributions}

O.Y.M.: conceptualization, investigation, visualization, writing — original draft, funding acquisition, supervision, study administration. A.A.A.-S.: formal analysis, methodology, validation, writing-review and editing. A.A.D.: writing-review and editing: investigation. W.Q.: investigation.

\section{Competing interests}

The authors declare no competing interests.

\section{Additional information}

Correspondence and requests for materials should be addressed to A.A.A.-S.

Reprints and permissions information is available at www.nature.com/reprints.

Publisher's note Springer Nature remains neutral with regard to jurisdictional claims in published maps and institutional affiliations.

Open Access This article is licensed under a Creative Commons Attribution 4.0 International License, which permits use, sharing, adaptation, distribution and reproduction in any medium or format, as long as you give appropriate credit to the original author(s) and the source, provide a link to the Creative Commons licence, and indicate if changes were made. The images or other third party material in this article are included in the article's Creative Commons licence, unless indicated otherwise in a credit line to the material. If material is not included in the article's Creative Commons licence and your intended use is not permitted by statutory regulation or exceeds the permitted use, you will need to obtain permission directly from the copyright holder. To view a copy of this licence, visit http://creativecommons.org/licenses/by/4.0/.

(C) The Author(s) 2021 